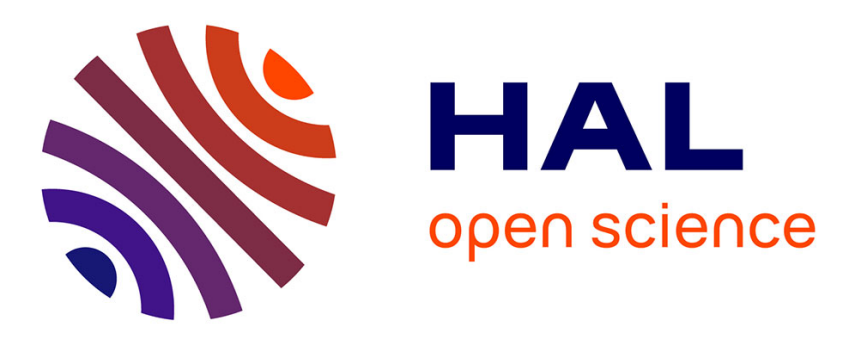

\title{
Identifying stationary phases in multivariate time series for highlighting behavioural modes and home range settlements
}

\author{
Rémi Patin, Marie-Pierre Etienne, Emilie Lebarbier, Simon \\ Chamaillé-Jammes, Simon Benhamou
}

\section{To cite this version:}

Rémi Patin, Marie-Pierre Etienne, Emilie Lebarbier, Simon Chamaillé-Jammes, Simon Benhamou. Identifying stationary phases in multivariate time series for highlighting behavioural modes and home range settlements. Journal of Animal Ecology, 2020, 89 (1), pp.44-56. 10.1111/1365-2656.13105 . hal-02311427v2

\author{
HAL Id: hal-02311427 \\ https://hal.science/hal-02311427v2
}

Submitted on 16 Nov 2020

HAL is a multi-disciplinary open access archive for the deposit and dissemination of scientific research documents, whether they are published or not. The documents may come from teaching and research institutions in France or abroad, or from public or private research centers.
L'archive ouverte pluridisciplinaire HAL, est destinée au dépôt et à la diffusion de documents scientifiques de niveau recherche, publiés ou non, émanant des établissements d'enseignement et de recherche français ou étrangers, des laboratoires publics ou privés. 


\title{
Identifying stationary phases in multivariate time series for highlighting behavioural modes and home range settlements
}

\author{
Rémi Patin $^{1}$ | Marie-Pierre Etienne ${ }^{2}$ | Emilie Lebarbier $^{3}$ | Simon Chamaillé-Jammes ${ }^{1,4,5}$ (D) | \\ Simon Benhamou ${ }^{1}$
}

${ }^{1}$ Centre d'Écologie Fonctionnelle et Évolutive, CNRS et Université de Montpellier, Montpellier, France

${ }^{2}$ Institut de recherche mathématique de Rennes, Université de Rennes, AgroCampusOuest, Rennes, France

${ }^{3}$ Mathématiques et Informatique Appliquées, Agroparistech, Paris, France

${ }^{4}$ LTSER France, Zone Atelier

'Hwange', Hwange National Park, Dete, Zimbabwe

${ }^{5}$ Department of Zoology \& Entomology, Mammal Research Institute, University of Pretoria, Pretoria, South Africa

\section{Correspondence}

Simon Benhamou

Email: simon.benhamou@cefe.cnrs.fr

Handling Editor: Garrett Street

\section{Abstract}

1. Recent advances in biologging open promising perspectives in the study of animal movements at numerous scales. It is now possible to record time series of animal locations and ancillary data (e.g. activity level derived from on-board accelerometers) over extended areas and long durations with a high spatial and temporal resolution. Such time series are often piecewise stationary, as the animal may alternate between different stationary phases (i.e. characterized by a specific mean and variance of some key parameter for limited periods). Identifying when these phases start and end is a critical first step to understand the dynamics of the underlying movement processes.

2. We introduce a new segmentation-clustering method we called segclust $2 \mathrm{~d}$ (available as a R package at cran.r-project.org/package=segclust2d). It can segment bivariate (or more generally multivariate) time series and possibly cluster the various segments obtained, corresponding to different phases assumed to be stationary. This method is easy to use, as it only requires specifying a minimum segment length (to prevent over-segmentation), based on biological rather than statistical considerations.

3. This method can be applied to bivariate piecewise time series of any nature. We focus here on two types of time series related to animal movement, corresponding to (a) at large scale, series of bivariate coordinates of relocations, to highlight temporary home ranges, and (b) at smaller scale, bivariate series derived from relocations data, such as speed and turning angle, to highlight different behavioural modes such as transit, feeding and resting.

4. Using computer simulations, we show that segclust2d can rival and even outperform previous, more complex methods, which were specifically developed to highlight changes of movement modes or home range shifts (based on hidden Markov and Ornstein-Uhlenbeck modelling), which, contrary to our method, usually require the user to provide relevant initial guesses to be efficient. Furthermore, we demonstrate it on actual examples involving a zebra's small-scale movements and an elephant's large-scale movements, to illustrate how various movement modes and home range shifts, respectively, can be identified.

\section{KEYWORDS}

area-concentrated searching, clustering, foraging, home range, migration, movement ecology, segmentation, transit 


\section{1 | INTRODUCTION}

Landscapes are spatially and temporally variable at various scales (Levin, 1992), and animals are expected to adjust their movements to the characteristics of their local environment, so as to maximize the time spent in profitable (or safe) habitats and minimize time in adverse ones (Pyke, 1978). Recent advances in biologging have made it possible to acquire time series of animal's locations, and possibly ancillary data such as activity level derived from on-board accelerometers, over extended areas and long durations with high spatial and temporal resolutions. Such locational time series, and the various series that can be derived from them to describe the movement behaviour (e.g. turning angle, speed), are therefore expected to be piecewise stationary, that is to present a specific mean and variance for limited periods corresponding to stationary phases, alternating with rapid transition phases corresponding to changes of areas or behaviours (in practice, a phase can be considered as being stationary when the partial means and variances obtained for its first and second halves or for its three thirds are not markedly different). Identifying these stationary phases is a prerequisite to determine the biologically relevant scales of movement (Benhamou, 2014). It is therefore of paramount importance in two types of movement studies:

\section{1 | Identifying behavioural modes}

Foragers are generally expected to alternate intensive (area-concentrated) searching mode, characterized by high tortuosity and low speed, and extensive searching (transit) mode, characterized by low tortuosity and high speed (see Dias, Granadeiro, \& Palmeirim, 2009, for contrasting examples). A number of discrete time methods have been developed to attempt to identify the different movement modes (see Gurarie et al. (2016) and Edelhoff, Signer, and Balkenhol (2016) for general reviews; see also Parton and Blackwell (2017) and Michelot and Blackwell (2019) for continuous-time approaches). As the alternation of searching modes should result in piecewise 'behavioural stationarity' when considering time series of some movement parameters, several segmentation approaches have been developed to identify behavioural modes by looking at breakpoints (i.e. rapid transitions between stationary phases; Barraquand \& Benhamou, 2008; Gurarie, Andrews, \& Laidre, 2009; Nams, 2014). A more sophisticated approach based on hidden Markov models (HMMs) has gained momentum in recent years. In this approach, the joint step lengths and turning angles calculated from successive relocations are categorized among a predefined number of different modes modelled as hidden states (Beyer, Morales, Murray, \& Fortin, 2013; Langrock et al., 2012; McClintock et al., 2012; Michelot, Langrock, \& Patterson, 2016; Morales, Haydon, Frair, Holsinger, \& Fryxell, 2004). However, the convergence of HMM may depend on the ability of the user to specify relevant initial state-dependent probability distribution parameters. Here, we aim at developing an alternative approach which does not require such a pre-specification. As most previous methods, it focuses on speed and tortuosity but deals with such variable through a penalized likelihood criterion. Furthermore, in addition to the spatiotemporal couple of metrics classically used in HMM-based segmentation (linear and angular speeds), we aim at testing other couples of metrics which should be more able to differentiate between the various behavioural modes by accounting for spatial and temporal effects separately (Benhamou \& Bovet, 1989), or which present more suitable statistical characteristics (Gurarie et al., 2009).

\section{2 | Identifying home range shifts}

The recently emerging question of piecewise 'locational stationarity' at the home range scale has been addressed in terms of movement scales (Benhamou, 2014), migration characteristics (Cagnacci et al., 2016; Naidoo, Preez, Stuart-Hill, Jago, \& Wegmann, 2012) and of within-season shifts (Couriot et al., 2018). Indeed, for an animal that exploits various temporary home ranges, the time series of relocations coordinates can be assumed to be stationary for a relatively long time (when the animal exploited the area where it established its temporary home range), then non-stationary for a relatively short time (when the animal left its home range until it established a new one) and so on. It is worth noting that a shift in home range does not necessarily involve a shift in mean location. It may also correspond to a change in variance if the animal enlarged or shrank its home range, for example due to a change of season (Monsarrat et al., 2013; Naidoo et al., 2012) or in reproductive status. Various methods have been proposed to detect home range shifts. The simple univariate approach based on the change in the beeline distance from a starting point (Bunnefeld et al., 2011) appears to be convenient in some cases but fully ignores movements leading the animal at a similar distance from the starting point but in another direction. More complex approaches rest on OrnsteinUhlenbeck (OU) modelling, following Blackwell (1997). Breed, Golson, and Tinker (2017) used a Bayesian framework to estimate the number of states of an underlying multistate OU process. In this way, they could directly infer the effect of covariates on the transition rate between ranges, but used a simple K-means algorithm to assign locations to the different states (corresponding to the different home ranges). Gurarie et al. (2017) developed an alternative approach by introducing a non-stationary state in the OU process to model the shifts in home range location. However, as it requires that all home range phases and shifts are explicitly modelled, this approach tends to become cumbersome when there are several shifts to consider. Furthermore, it may require truly relevant initial guesses to correctly detect small shifts. We therefore aim at developing an alternative approach that could be more efficient than an $\mathrm{OU}$-based approach to detect home range shifts and simpler to use. Additionally, as detecting shifts in behavioural modes and in home ranges settlements are conceptually similar, we focused on a generic approach that can be applied to both types of studies. 


\section{3 | Introducing a new method}

In this paper, we introduce a new method, called segclust2d, able to segment a bivariate (and more generally multivariate) time series and to cluster similar segments (corresponding to stationary phases) in a common class (corresponding to a given state) if desired. We demonstrate that this method, which is easy to use, can successfully identify stationary phases corresponding to temporary home ranges when based on bivariate locational time series, as well as movement modes when based on bivariate time series of metrics such as speed and tortuosity. It thus offers an efficient and user-friendly alternative to previous, more complex, approaches. Furthermore, as this model applies to multivariate piecewise stationary time series based on any kind of metrics, it can integrate additional time series of ancillary data (e.g. activity level derived from on-board accelerometers) for a better segmentation-clustering of movement data.

\section{2 | MATERIALS AND METHODS}

\section{1 | Statistical models and parameter estimation}

\subsection{1 | General principle}

Consider a multivariate time series composed of $C$ components (each corresponding to a univariate time series). These components are assumed to be statistically independent and should be normalized if they are of different nature, so as to have the same weight in subsequent procedures. The time series is assumed to be piecewise stationary, that is to be made of an unknown number of stationary phases. In a given phase, the values taken by any of the $C$ components are assumed to be independent of each other. One needs a reliable statistical model to detect and locate these phases, and possibly to cluster them when they are assumed to be the expressions of a limited number of unobserved states of the underlying process (e.g. behavioural modes). Likelihood-based segmentation methods provide a suitable statistical framework to detect changes of phases but raise two main issues from a statistical and algorithmical point of view: (a) determining the optimal number of segments and (b) for a given number of segments, finding the optimal segmentation, that is determining the locations of the starting/ending points of the segments (called breakpoints). The latter reduces to a well-known discrete optimization problem solved using a dynamic programming algorithm introduced by Bellman (1954; for a recent example, see Rigaill, 2015). For a time series of $n$ values that can potentially be cut at any point in $K$ segments, the dynamic programming algorithm reaches the exact maximum-likelihood solution with a complexity in $O\left(n^{2} K\right)$, drastically smaller than the complexity in $O\left(n^{K}\right)$ involved by a force brute algorithm exploring the whole segmentation space. We will first introduce the models and the estimation procedure to optimally segment a multivariate signal for a predefined number of segments $K$ and possibly (if clustering is required) a predefined number of states $M$. Afterwards, we will show how the optimal number of segments and possibly of clusters can be found based on a penalized likelihood criterion. Our approach is based on
Lavielle (2005)'s segmentation method of univariate signals and its extension by Picard, Robin, Lebarbier, and Daudin (2007) to segment and cluster DNA sequences without assuming any kind of distribution for the segment lengths, such as a geometric distribution as HMM implicitly do (Karlin \& Taylor, 1975).

\subsection{2 | Optimal segmentation in $K$ segments, with optional clustering in $\mathrm{M}$ states}

Assume that there are $K$ stationary phases in a multivariate time series with total length $n$. A stationary phase corresponds to a segment. On each segment and for each of the $C$ components (labelled 1 to $C$ ), the series is assumed to be a sequence of random variables sharing the exact same distribution, in particular the same mean and the same variance. As soon as one of these parameters changes, a new segment starts. Formally, the $C$ components within a given segment $k \in[1, \ldots, K]$ starting at time $t=t_{k-1}+1$ and ending at time $t=t_{k}$ (with $t_{0}=0$ and $t_{K}=n$ by convention) are modelled as sequences of Gaussian independent random variables $Y(t)=\left(Y_{1}(t)\right.$, $\left.\ldots, Y_{C}(t)\right)^{\top}$ for $t=1, \ldots, n$.

The segmentation-only model (no clustering) is written simply as

$\mathbf{Y}\left(t \mid t \in\left[t_{k-1}+1, t_{k}\right]\right) \sim N\left(\mu_{k}, \sigma_{k}^{2}\right)$, with $\mu_{k}=\left(\begin{array}{c}\mu_{k, 1} \\ \vdots \\ \mu_{k, C}\end{array}\right)$ and $\sigma_{k}^{2}=\left(\begin{array}{ccc}\sigma_{k, 1}^{2} & 0 & 0 \\ 0 & \ddots & 0 \\ 0 & 0 & \sigma_{k, C}^{2}\end{array}\right)$

where $\boldsymbol{\mu}_{k}$ and $\boldsymbol{\sigma}_{k}^{2}$ are the mean vector and the variance matrix for data in segment $k$. As the model parameters to be estimated vary independently between segments, dynamic programming can be used to segment the multivariate signal at best in $K$ segments. Its application is straightforward in this case, as it relies on the log-likelihood of each segment, which is simply equal to the sum of the log-likelihoods of the $C$ components.

In the segmentation-clustering model, a state $m$, among $M$ possible states, is assigned to every segment. Segments that are classified in state $m$ are all assumed to share the same mean vector $\boldsymbol{\mu}_{m}$ and the same variance matrix $\boldsymbol{\sigma}_{m}^{2}$. More formally, let $S_{k}$, denotes the state of the segment $k$, with $k=1, \ldots, K . S_{k}$ is a latent random variable taking values in $[1, \ldots, M]$. It is modelled through a multinomial distribution of parameters $\pi=\left(\pi_{m}\right)$ with $m=1, \ldots, M$, where $\pi_{m}$ corresponds to the probability for a segment to belong to state $m$. The segmentation-clustering model can therefore be written as

$\mathbf{Y}\left(t \mid t \in\left[t_{k-1}+1, t_{k}\right], S_{k}=m\right) \sim N\left(\mu_{m}, \boldsymbol{\sigma}_{m}^{2}\right)$, with $\boldsymbol{\mu}_{m}=\left(\begin{array}{c}\mu_{m, 1} \\ \vdots \\ \mu_{m, C}\end{array}\right)$ and $\boldsymbol{\sigma}_{m}^{2}=\left(\begin{array}{ccc}\sigma_{m, 1}^{2} & 0 & 0 \\ 0 & \ddots & 0 \\ 0 & 0 & \sigma_{m, C}^{2}\end{array}\right)$

As the parameters $\left(\pi_{m}, \boldsymbol{\mu}_{m}, \boldsymbol{\sigma}_{m}^{2}\right)$ that characterize any state $m$ are unknown and are to be estimated, resulting in a mixture distribution where segments are linked in terms of parameters, the 
optimal segmentation cannot anymore be obtained using dynamic programming alone. Following Picard et al. (2007), we designed the following two-step procedure, which is iterated up to convergence.

1. Given a set of parameters $\left(\pi_{m}, \boldsymbol{\mu}_{m}, \boldsymbol{\sigma}_{m}^{2}\right)$ with $m=1, \ldots, M$, the best segmentation in $K$ segments is obtained using dynamic programming.

2. Given this segmentation, the values of parameters are estimated using an expectation-maximization algorithm which is commonly used in latent variable modelling (Dempster, Laird, \& Rubin, 1977).

By mixing dynamic programming and expectation-maximization through this iterative procedure, segmentation and clustering processes work jointly (rather than the latter after the former) leading to the optimal segmentation given $K$ and $M$. An additional procedure for initializing the EM algorithm is automatically performed to solve possible convergence issues (see details in Supporting information 1). Note also that this method has to be applied separately to data coming from different individuals because it is not designed to perform a joint estimation of parameters for disjoint time series (i.e. concerning different individuals).

\subsection{3 | Finding the optimal numbers of segments and states}

For both models (segmentation-only and segmentation-clustering), a minimum segment length $L_{\text {min }}$ (>5 records to warrant a sufficiently reliable estimation of the parameters) has to be set not only to speed up the algorithm, but also, more fundamentally, to prevent over-segmenting, based on biological considerations. For example, setting $L_{\text {min }}$ to a value of a few weeks when analysing locational time series will prevent the algorithm from considering an area exploited only for a few days, corresponding to foray outside the usual home range or to stopover during migration, as a distinct home range. Similarly, setting $L_{\min }$ to a value long enough (depending on the species) when looking for changes of behavioural modes will force the algorithm to assign a given behavioural bout to a given mode even when it is interspersed by ephemeral events related to another behaviour (e.g. a long transit with opportunistic short feeding events on the move will be considered as a single transit phase). The likelihood is calculated for all numbers of segments $K<0.75 n / L_{\text {min }}$ (larger values of $K$ may generate inconsistent results), and for any number of states $M$ $(<K)$ one wishes to consider if clustering has to be involved. In the latter case, the optimal values of $K$ and $M$ are determined as those that maximize a Bayesian information criterion (BIC; Schwarz, 1978)based penalized likelihood (Supporting information 1). However, as it will be shown in the Results section, it is usually preferable to set the number of states $M$ based on biologically relevant grounds than to let the model selection criterion determine an optimal number of states based on a statistical basis (see also Pohle, Langrock, Beest, \& Schmidt, 2017). When no clustering is involved, the optimal number of segments $K$ is based in agreement with Lavielle (2005) on maximizing a K-penalized likelihood curve (Supporting information 1).

\section{2 | Computer simulations}

We run simulations to assess the ability of our approach to detect home range shifts and changes of behavioural modes from bivariate time series, and to compare it with that of other (OUM-based and HMM-based) methods. For each set of parameters of each type of simulation, we simulated 100 replicates. Distances are expressed in arbitrary unit length $\mathrm{u}$.

\subsection{1 | Home range shifts}

For simplicity, the animal was assumed to behave as a central place forager. We simulated its fine-scale movement as a central-place biased correlated random walk involving a differential klinokinetic mechanism, which results in a probability of presence decreasing exponentially with the distance $D$ to the central place (Benhamou, 1989): at each time step, the animal turns by an angle $\alpha_{i}$ drawn from a wrapped Gaussian distribution with a null mean and standard deviation $s_{i}=s_{0}\left[1+b\left(D_{i}-D_{i-1}\right)\right]$, with $b=0.5$ radian/u, and progresses by 1 unit length $(1 \mathrm{u})$ in the new direction. The directional bias is generated by the modulation of $s_{i}$ (in the range $\left.\left[0.5 s_{0}, 1.5 s_{0}\right]\right)$, which leads the animal to experience a higher probability to reverse its moving direction when its moves away of $\left(D_{i}>D_{i-1}\right)$ than towards $\left(D_{i}<D_{i-1}\right)$ the central place. In a batch of simulations, $s_{0}$ was set to 0.5 radian, and the central place was first set at a given location for the first 10,000 time steps (phase 1), then shifted to another location by $85 \mathrm{u}(60 \mathrm{u}$ in both $X$ and $Y$ ) for 10,000 additional time steps (phase 2), resulting in disjoint home ranges, and then shifted to a third location by $28 \mathrm{u}$ ( $20 \mathrm{u}$ in both $X$ and $Y$ ) for 10,000 additional time steps (phase 3 ), resulting in overlapping home ranges. In another batch of simulations, the central place remained at the same location for the 30,000 time steps, but $s_{0}$ was set to 0.7 radian for time steps 10,001-20,000 (phase 2) and to 0.5 radian otherwise (phases 1 and 3), involving a transitory enlargement of the home range. We finally subsampled the data sets by keeping one in every 60 locations. The home range phases were then defined by 166 locations each, with low serial correlation, and were thus similar to actual datasets that are commonly used in home range studies. Note that in our approach that focuses on the contrast between the stationary phases, the actual lengths of these phases do not matter, provided they are longer than $L_{\min }$.

\subsection{2 | Changes of behavioural modes}

We simulated a random search movement as a correlated random walk where three types of activity-immobility (resting or standing), intensive (area-concentrated) searching and extensive searching (transit)-alternate, each one lasting 20 time steps, this 60-step subseries being repeated five times. The step lengths $L_{i}$ were drawn from a log-normal distribution with a mean equal to $0.5 \mathrm{u}$ in the intensive mode or $1.0 \mathrm{u}$ in the extensive mode, and with a standard deviation equal to $1 / 10$ th of the mean in both modes. Turning angles $\alpha_{i}$ were drawn from a wrapped Gaussian distribution with a null mean and a standard deviation equal to 0.4 radian in the intensive mode or 0.3 radian in the extensive mode. To mimic possible factors (e.g. GPS 
recording noise) that can blur the contrast between the modes, the locations obtained in this way, as well those obtained for immobility phases, were submitted to bivariate Gaussian random noise with a null mean and various standard deviations $\zeta$. Note that in order to assess the ability of a method to segment a behavioural time series, the precise movement rules used in simulations are not important. What really matters is the contrast between the different phases: with a high contrast, all methods should work well, whereas with a low contrast, all methods should fail, whatever the movement rules considered. In the results section, we will present the results obtained with a standard deviation of the noise $\zeta$ set to $0.2,0.3$ and 0.4 , involving a high, moderate and low contrast, respectively, between the three modes.

\section{3 | Metrics}

For identifying home range shifts, the two signal components considered are orthonormal Cartesian coordinates $\left(x_{i}, y_{i}\right)$ of locations (GPS locations expressed in decimal degrees as longitude and latitude therefore require to be transformed in terms of easting and northing through a classical projection such as UTM). For identifying behavioural modes, the two components usually considered in HMM-based approaches are the classical metrics corresponding to the step lengths $L_{i}$ and the turning angles $\alpha_{i}$, computed from locations recorded at constant time intervals $\Delta t$, and therefore acting as proxies for linear $\left(L_{i} / \Delta t\right)$ and angular $\left(\alpha_{i} / \Delta t\right)$ speeds, respectively. We used such metrics for comparative purpose, but we also tested some variants, assumed to improve the contrast between the different modes. We computed the linear speed as $\left(L_{i}+L_{i+1}\right) /(2 \Delta t)$. Although this basic smoothing introduces some serial correlation ( $\rho=.5$ ) which is not taken into account in our model, it should result in a less noisy signal. Furthermore, angular speed may show faded changes with searching modes because the intensive mode usually involves both a decrease in linear speed and an increase in path tortuosity but angular speed mechanically increases with both of them (Barraquand \& Benhamou, 2008; Benhamou \& Bovet, 1989). We therefore computed turning angles $\alpha_{i}^{*}$ based on a constant step length $r$ rather than at constant time interval. For this purpose, each location $X_{i}=\left(X_{i}, Y_{i}\right)$ is considered the centre of a virtual circle with radius $r$, and the entrance and exit locations $\mathbf{P}_{\text {en }}$ and $\mathbf{P}_{\mathrm{ex}}$ are determined through linear interpolation (Supporting information 2). The turning angle $\alpha_{i}^{*}$ is then computed in $[-\pi, \pi]$ as the angular deviation between vectors $\mathbf{P}_{\text {en }} \rightarrow \mathbf{X}_{i}$ and $\mathbf{X}_{i} \rightarrow \mathbf{P}_{\text {ex }}$ (both with length r) rather than vectors $X_{i-1} \rightarrow X_{i}$ (with length $L_{i}$ ) and $X_{i} \rightarrow X_{i+1}$ (with length $L_{i+1}$ ) as done to compute $\alpha_{i}$. When both $L_{i}$ and $L_{i+1}$ are larger than $r$, one gets $\alpha_{i}^{*}=\alpha_{i}$, whereas $\left|\alpha_{i}^{*}\right|$ tends, on average, to be larger (random search paths) or smaller (directed paths) than $\left|\alpha_{i}\right|$ when $r$ is larger. In our simulations, we noticed that using a radius larger than the median of the overall step length distribution tends to improve the discrimination between the fast and slow movement modes but to worsen the discrimination between the slow movement mode and the immobility mode. We therefore chose to set $r$ to the median of the step length distribution and accordingly used this value in all our analyses. We also tested the two orthogonal signals provided by the 'persistence speed' $L_{i+1} \cos \left(\alpha_{i}\right) / \Delta t$ and 'turning speed' $L_{i+1} \sin \left(\alpha_{i}\right) / \Delta t$ (Gloaguen et al., 2015; Gurarie et al., 2009).

\subsection{Practical implementation of the method}

Both segclust2d procedures (segmentation-only and segmentationclustering) have been currently implemented for bivariate times series (the case we considered in this paper) in an $\mathrm{R}$ package (available at cran.r-project.org/package=segclust2d). An integrated module makes it possible to derive the various movement variables mentioned in this paper from locations data. Because our approach requires large amounts of computer memory, it cannot deal with too long time series (>10,000 values) on small desktop computers. It is worth noting, however, that, even in absence of any memory constraints, it is usually not a good practice to attempt to directly segment very long series, which encompass both very large-scale phenomena (thanks to their large extent) and very small-scale phenomena (thanks to their high resolution). Indeed, small-scale data are usually not relevant for analysing large-scale patterns and therefore act more as noise than as information in this context. For these reasons, some subsampling (thinning) is automatically performed by segclust $2 \mathrm{~d}$ when necessary. Thus, in our home range simulations, keeping one in every 60 locations makes it possible to drastically shorten the time series by eliminating fine-scale movements (klinokinetic process), which are characterized by a high level of serial correlation in location and in direction. Such details are clearly not relevant for the question of home range shifts, where only the overall phase-dependent mean and/or the variance of locations matter (accordingly, our approach ignores serial correlations occurring in any stationary phase). Conversely, for fine-scale movement studies, the characteristics of the environment are liable to change (e.g. due to seasonal variations) when considering a time-series running over an extended duration, possibly leading to change the characteristics of the behavioural classes expected. It appears therefore preferable to consider the various periods (e.g. seasons) separately rather than to attempt to deal with the long time series as a whole. Finally, note that the time series to be analysed are assumed to be regular (no gaps). Nevertheless, some gaps in location data do not matter when the metrics used directly correspond to northing and easting, as they will not change their mean and variance. In contrast, for derived metrics such as speed or turning angle, the occurrence of missing locations may result in noticeable changes of the values obtained and is therefore likely to bias the segmentation-clustering process.

\section{3 | RESULTS}

\section{1 | Identifying home range shifts}

\subsection{1 | Simulated movements}

Figure 1 shows an example where the centre of the home range was shifted (by $85 \mathrm{u}$ between phases 1 and 2, and by $28 \mathrm{u}$ between phases 2 and 3), and an another example where the home range was enlarged during the phase 2 with respect to phases 1 and 3 . The 


\section{(a) Shifts in mean location: path}

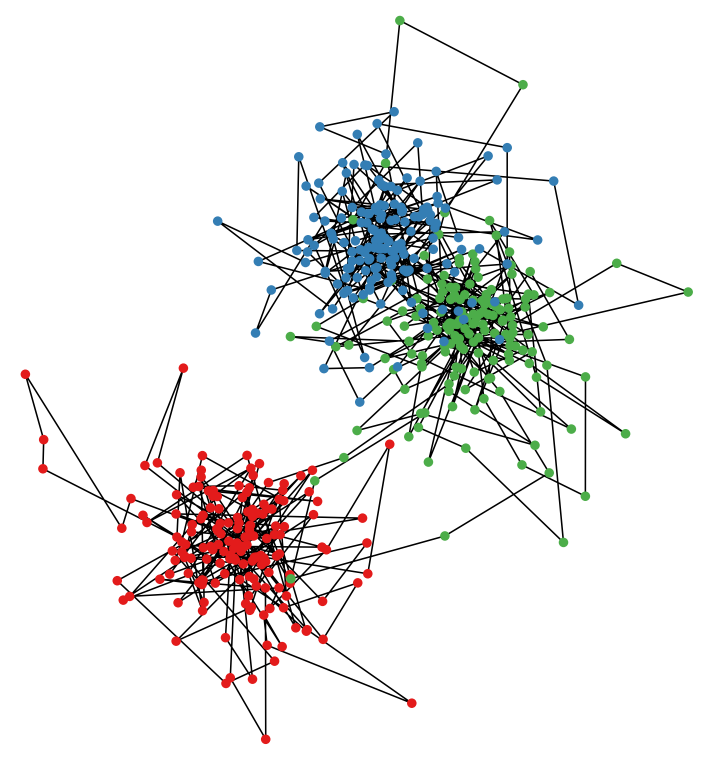

(c) Shifts in mean location: time-series

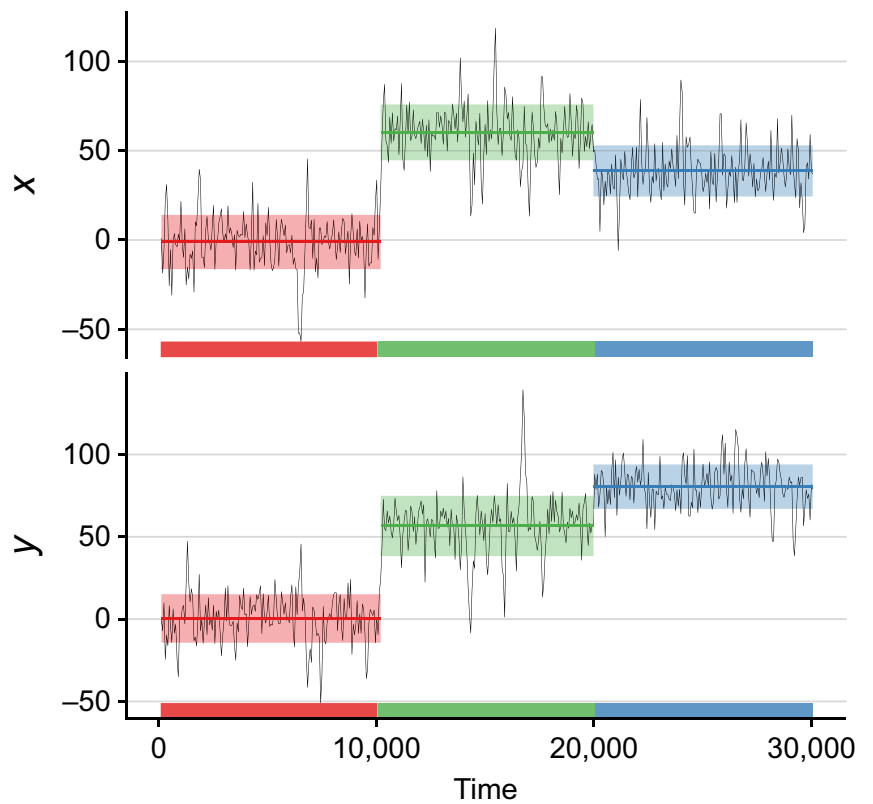

(b) Shifts in variance: path

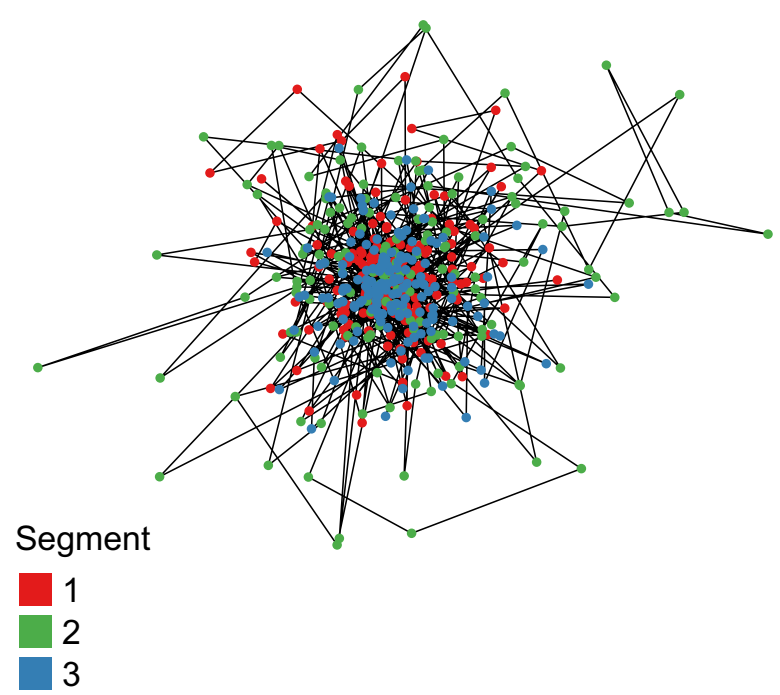

(d) Shifts in variance: time-series
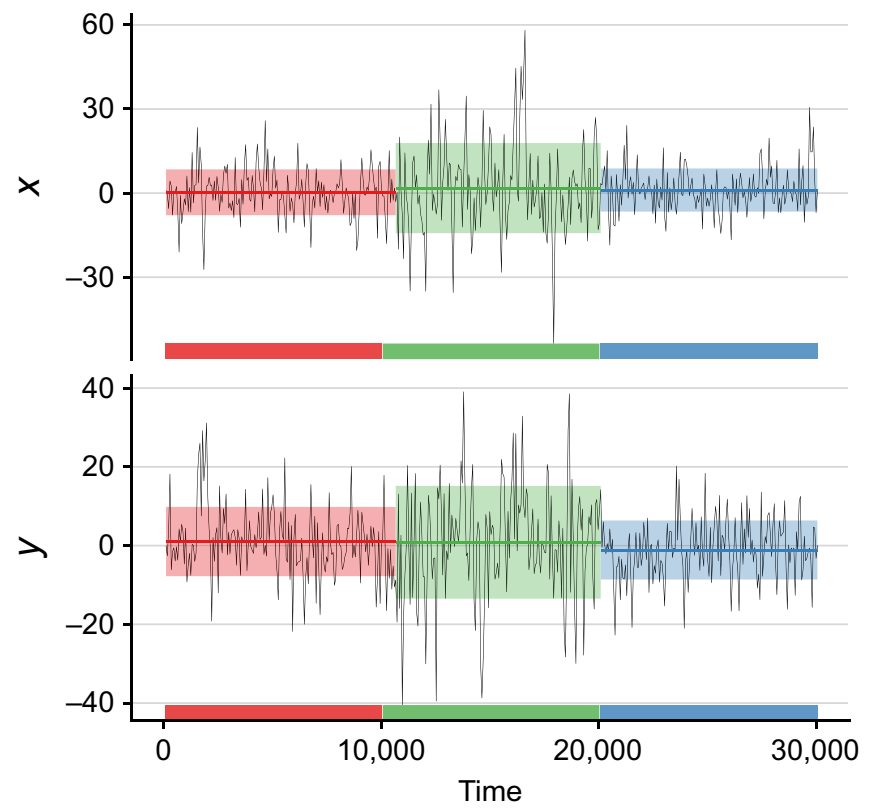

FIGURE 1 Examples of application of the segclus2d/segmentation-only procedure to highlight home range phases and shifts in simulated movements. Top panels show the simulated paths (after $1 / 60$ subsampling) corresponding to three home range phases (two shifts), either in mean location (a) or in variance (b). The corresponding time series for both location coordinates ( $x, y)$ are presented in panels (c) and (d), respectively. The horizontal colour bars running along the time axis show the true occurrences of the three phases, whereas the coloured bands appearing over the $x$ and $y$ signals show their occurrences as estimated using the segclust $2 \mathrm{~d} /$ segmentation-only method with $L_{\text {min }}=45$ locations (corresponding to 2,700 time steps because of the $1 / 60$ subsampling) and provide the estimated mean (plain horizontal line running in the middle of the band) $\pm S D$ (band width) for each segment separately

segmentation-only procedure correctly determined that the actual number of phases was equal to 3 in 98 out of the 100 replicates involving shifts in mean location (i.e. central place), and in 88 out of the 100 replicates involving shifts in variance (i.e. change in home range size). In these cases, the timing of the various shifts was also correctly determined (mean $\pm S D=10,152 \pm 79$ and 20,092 \pm 188 time steps for the $85-\mathrm{u}$ and $28-\mathrm{u}$ shifts in mean location, respectively; mean $\pm S D=10,035 \pm 1,184$ and 19,942 $\pm 1,314$ time steps for the first and second shifts in variance of the same amplitude). In contrast, the OU-based algorithm 'marcher', which was specifically developed by Gurarie et al. (2017) to identify home range shifts in mean location, requires that the number of shifts has been specified and is unable to detect shifts in variance. Without initial information about the possible timing of the shifts, this method relies on a simple $k$-means procedure 
(a)

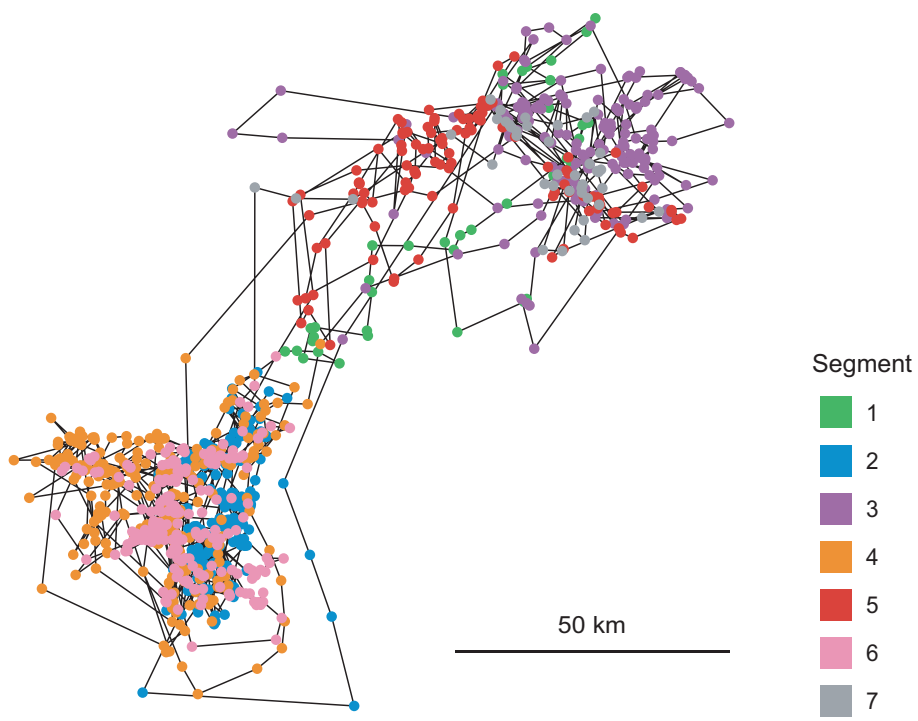

(b)
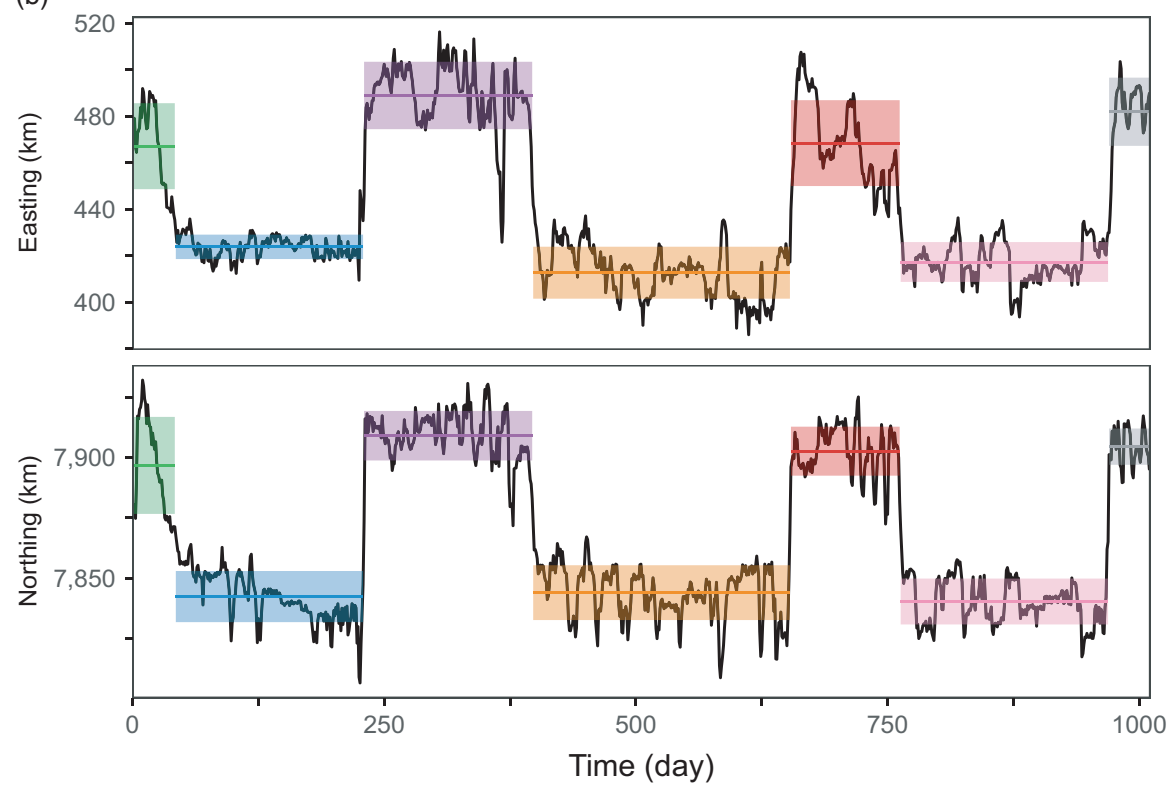

FIGURE 2 Example of application of the segclust $2 d /$ segmentation-only procedure to highlight home range phases and shifts in an African elephant's movement recorded over 1,000 days. (a) Rough path representation obtained by linking the locations subsampled so as to keep a single GPS location per day; (b) corresponding time series of locations coordinates (easting and northing). The coloured bands appearing over the time series show the estimated mean (plain horizontal line running in the middle of the band) $\pm S D$ (band width) of each of the seven segments obtained using the segmentation-only method with $L_{\min }=30$ days to get initial guesses, and was then only able to detect the large shift in location (mean $\pm S D=10,152 \pm 937$ time steps; the small one occurred anywhere unpredictably between 15,000 and 30,000 time steps, based only on 75 replicates, as the algorithm failed to provide any result for 25 replicates). However, the 'marcher' algorithm proved able to correctly detect the two shifts in mean location (mean $\pm S D=10,152 \pm 82$ and 19,967 \pm 239 time steps for the 85-u and 28-u shifts in mean location, respectively) when the actual home range centres and shift dates were provided as truly relevant initial guesses.

\subsection{2 | Illustrative example}

We used the GPS track of an African Elephant (Loxodonta africana), recorded for $>2.5$ years to illustrate the way the segclust $2 \mathrm{~d} /$ segmentation-only procedure can identify home ranging phases and shifts. The whole time series of easting and northing coordinates appeared to be stationary and thus corresponds to a large multiannual (possibly lifetime) home range. At smaller scale, it also appeared to be piecewise stationary. It can therefore be segmented to highlight temporary (possibly seasonal) smaller home ranges and the shifts between them (Figure 2). However, some of the phases so highlighted are clearly non-stationary. In particular, segments 1 (days 1-46) and 5 (days 655-761) correspond mainly to a slow south-westward migration between the two core areas of the multiannual home range. Segment 2 also corresponds to a non-stationary, migratory (southwards moving) phase, which went through an area used as a temporary home range during segments 4 and 6 . This indicates that a same area can be used in different ways at different periods.

\section{2 | Identifying behavioural modes}

\subsection{1 | Simulated movements}

An example of a path with three behavioural modes (extensive searching, intensive searching and resting) is shown in Figure 3 with the corresponding time series in terms of absolute turning angle 
(a)

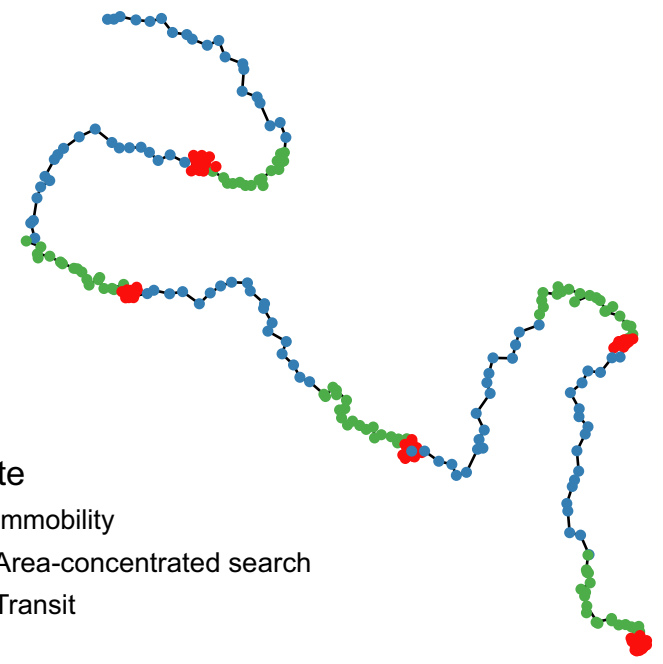

(b)
Number of clusters

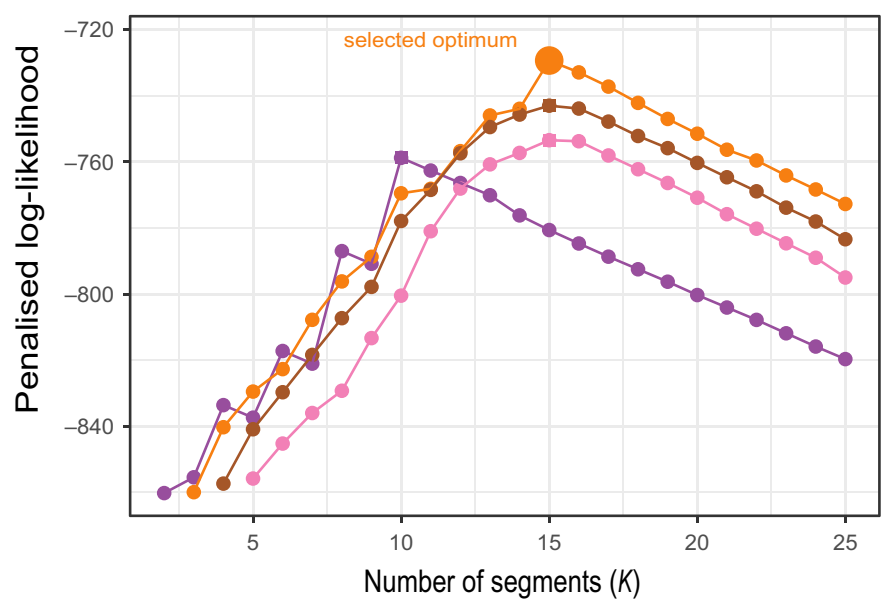

(c)
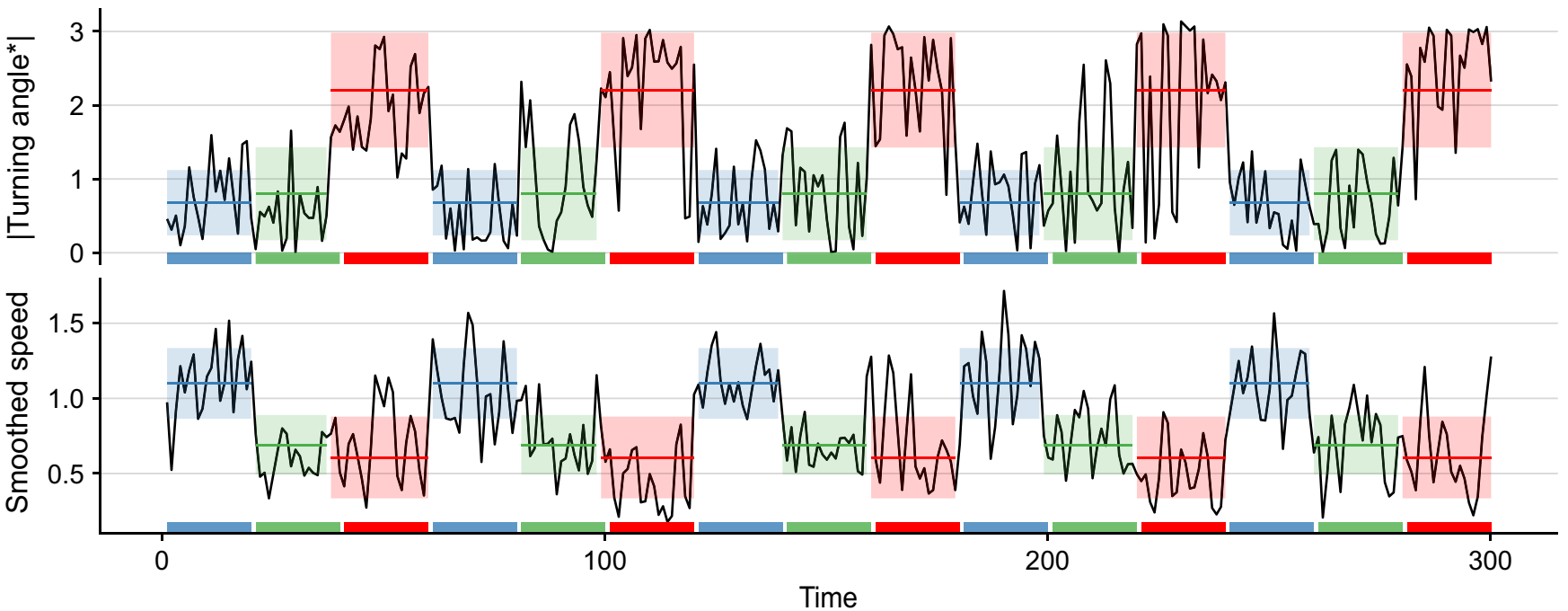

FIGURE 3 Example of application of the segclust2d/segmentation-clustering procedure to highlight behavioural changes in a simulated movement. (a) Simulated path as a composite correlated random walk, incorporating an additional noise with standard deviation $\zeta=0.3 \mathrm{u}$ that affects locations; (b) determination using BIC-based penalized likelihood of the most likely numbers of states $(M=3)$ and segments $(K=15)$ (big orange dot), and of the most likely number of segments for the other three numbers of states considered (large squares at the top of the curves); (c) corresponding time series in terms of absolute turning angle computed with a constant step length, $\left|\alpha_{i}^{*}\right|$, and smoothed speed, $\left(L_{i}+L_{i+1}\right) /(2 \Delta t)$, segmented with $L_{\min }=10$ and $M=3$; the coloured bands appearing over the two time series show the estimated occurrences and mean (plain horizontal line running in the middle of the band) $\pm S D$ (band width) for each of the three movement modes, whereas the horizontal colour bars running along the time axis show the true occurrences of these modes

$\left|\alpha_{i}^{*}\right|$ and smoothed speed $\left(L_{i}+L_{i+1}\right) /(2 \Delta t)$. In this example, the segclust $2 \mathrm{~d} /$ segmentation-clustering procedure appears able to detect the true number of modes $(M=3)$ and to attribute almost all locations to the right mode. We compared our method with a HMMbased method specifically designed to deal with movement data (McClintock \& Michelot, 2018; Michelot et al., 2016) when the true number of modes has been specified. The results obtained from 100 replicates showed that our procedure rivals with the HMMbased method although the latter was helped by initial state-dependent probability distribution parameters which were tuned to their true values for each behavioural state (Figure 4 with medium noise level $\zeta=0.3)$. With a low noise level $(\zeta<0.2)$, an excellent fit was obtained with all methods and metrics considered, whereas with a high noise level $(\zeta>0.4)$, the percentage of correct state assignment became closer to the value expected for a random assignment (33\%; see Supporting information 3.1 results with $\zeta=0.2$ and $\zeta=0.4)$. It also appeared that the angular $\left(\alpha_{i} / \Delta t\right)$ and linear $\left(L_{i} /\right.$ $\Delta t)$ speeds are suitable metrics for detecting behavioural changes with HMM only when the noise is not too high. With a high noise level, better results were obtained with both methods when using any other couple of metrics. The best fits were obtained with turning angle $\alpha_{i}^{*}$ or it absolute value $\left|\alpha_{i}^{*}\right|$ and smoothed speed. When the true number of modes is unknown, our method can also estimate this number as the most likely number of clusters, but the fraction of correct estimates is too low to consider the result as reliable (Supporting information 3.2). 


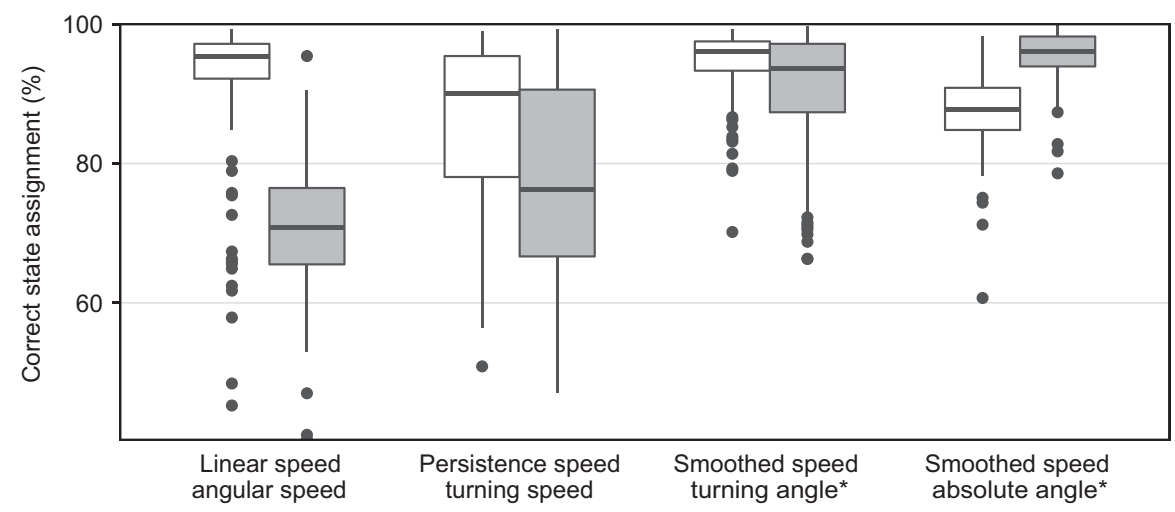

FIGURE 4 Comparative performances of the segclust2d/segmentation-clustering procedure versus a HMM-based method for highlighting behavioural changes. The boxplots show the proportion of correct state assignments, obtained for various bivariate signals when the true number of states is known $(M=3)$, as estimated from 100 replicates simulated with the same parameters as to the one illustrated in Figure 3 (noise $\zeta=0.3$ ). The star $\left({ }^{*}\right)$ indicates turning angles computed with a constant step length, in terms of arithmetic $\left(\alpha_{i}^{*}\right)$ or absolute $\left(\left|\alpha_{i}^{*}\right|\right)$ values. The white boxplots show the results obtained with hidden Markov model-based R package momentuHMM (McClintock \& Michelot, 2018), with initial state-dependent probability distribution parameters tuned to their true values for the different states (using the following distributions: Gaussian for persistence and turning speeds, $L_{i+1} \cos \left(\alpha_{i}\right) / \Delta t$ and $L_{i+1} \sin \left(\alpha_{i}\right) / \Delta t$, wrapped Cauchy for angular speed $\alpha_{i} / \Delta$ t and turning angle $\alpha_{i}^{*}$, Weibull for linear speed, $L_{i} / \Delta t$, smooth speed, $\left(L_{i}+L_{i+1}\right) /(2 \Delta t)$, and absolute value of turning angle $\left.\mid \alpha_{i}^{*}\right)$. The grey boxplots show the results obtained using the segclust $2 \mathrm{~d} /$ segmentation-clustering procedure with $L_{\min }=10$

\subsection{2 | Illustrative example}

We used a 24-hr GPS track of a plains zebra (Equus quagga) to illustrate the way the segclust $2 \mathrm{~d} /$ segmentation-clustering procedure can identify the occurrences of the various movement modes (Figure 5). Although, in this example, the most likely number of modes was estimated to be five, we present the segmentation obtained when setting this number to three, assuming that the biologically relevant modes should be resting (or any other non-moving behaviour such as standing), feeding and transiting (the other two modes detected by our procedure when using five modes were assumed to correspond to mixed behaviours).

\section{4 | DISCUSSION}

We showed that the method we proposed, segclust2d, which extends the methods developed by Lavielle (2005) and Picard et al. (2007) to multivariate time series, makes it possible to reliably detect two types of change that are of key importance when studying free-ranging animal movements: home range shifts, based on bivariate time series of location coordinates (segmentation-only procedure), and changes of behavioural modes, based on bivariate time series of turning angles and speed (segmentation-clustering procedure). In any case, this new method is straightforward to tune: the user has just to set the minimum segment length $\left(L_{\min }\right)$ to a biologically relevant value. Nevertheless, it proved to work at least as well as, and often better than, other recent methods specifically designed to deal with either home range shifts (Gurarie et al., 2017) or changes of behavioural modes (McClintock \& Michelot, 2018; Michelot et al., 2016).
Gurarie et al. (2017) developed an OU-based method to identify home range shifts in mean location. Using computer simulations, we compared this approach, as implemented in Gurarie et al.'s 'marcher' algorithm, with segclutst2d/segmentation-only. Both methods are well able to detect large shifts in mean location. However, our method is also able to detect small shifts in mean location, whereas Gurarie et al.'s method requires a priori information on the actual mean locations and the shifts dates to correctly detect them, although this is precisely in this case that such information is usually lacking (i.e. they can hardly be guesstimated from visual inspection of the data). Furthermore, contrary to Gurarie et al.'s method, which can deal only with a few number of shifts which have to be specified in advance, our method can work with any number of shifts and is able to correctly estimate this number by itself in most cases. It is also able to reveal changes in home range size. Yet, to be efficient, our method does not require any initial guess. It simply requires specifying a minimum length $\left(L_{\text {min }}\right)$ for stationary phase to be called a temporary home range, shorter phases being assumed to correspond to transitory exploitations of restricted areas rather than to home ranges. However, whereas our method considers migrations as simple breakpoints, Gurarie et al.'s method can estimate the duration of migrations.

The elephant we considered in our illustrative example tended to move back and forth between two main areas. This kind of space use is common in migrating birds that commute between reproductive and wintering home ranges. However, there are numerous studies showing more complex patterns, with an animal setting several distinct temporary home ranges successively (Benhamou, 2014; Cagnacci et al., 2016; Couriot et al., 2018; Naidoo et al., 2012). The segmentation of a long piecewise locational time series in phases corresponding to temporary home ranges opens promising perspectives 
(a)

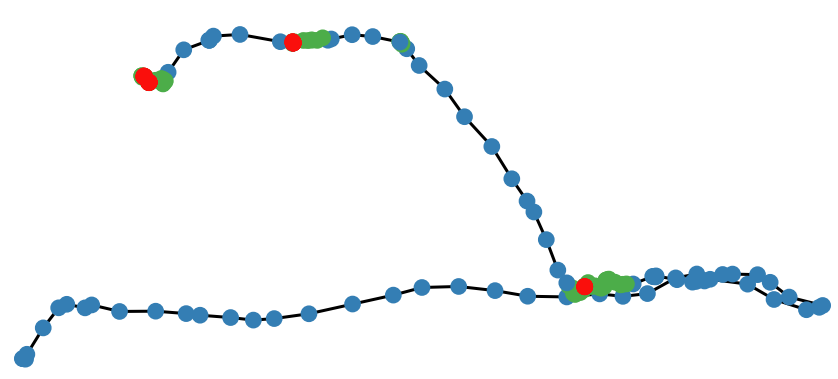

Behavioural mode

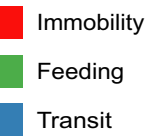

Number of clusters $\begin{array}{ccccc}- & - & - & - & -\end{array}$

(b)

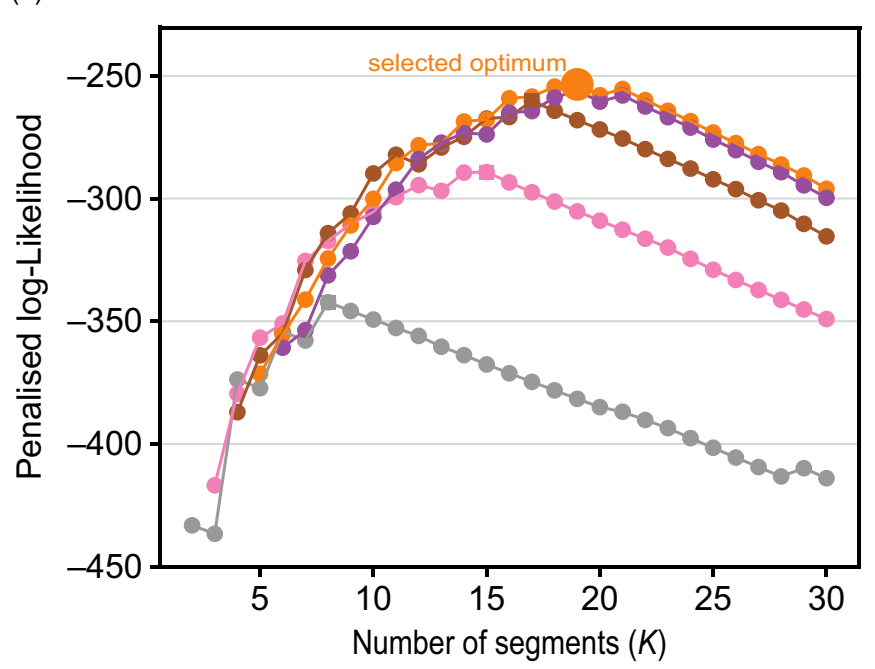

(c)
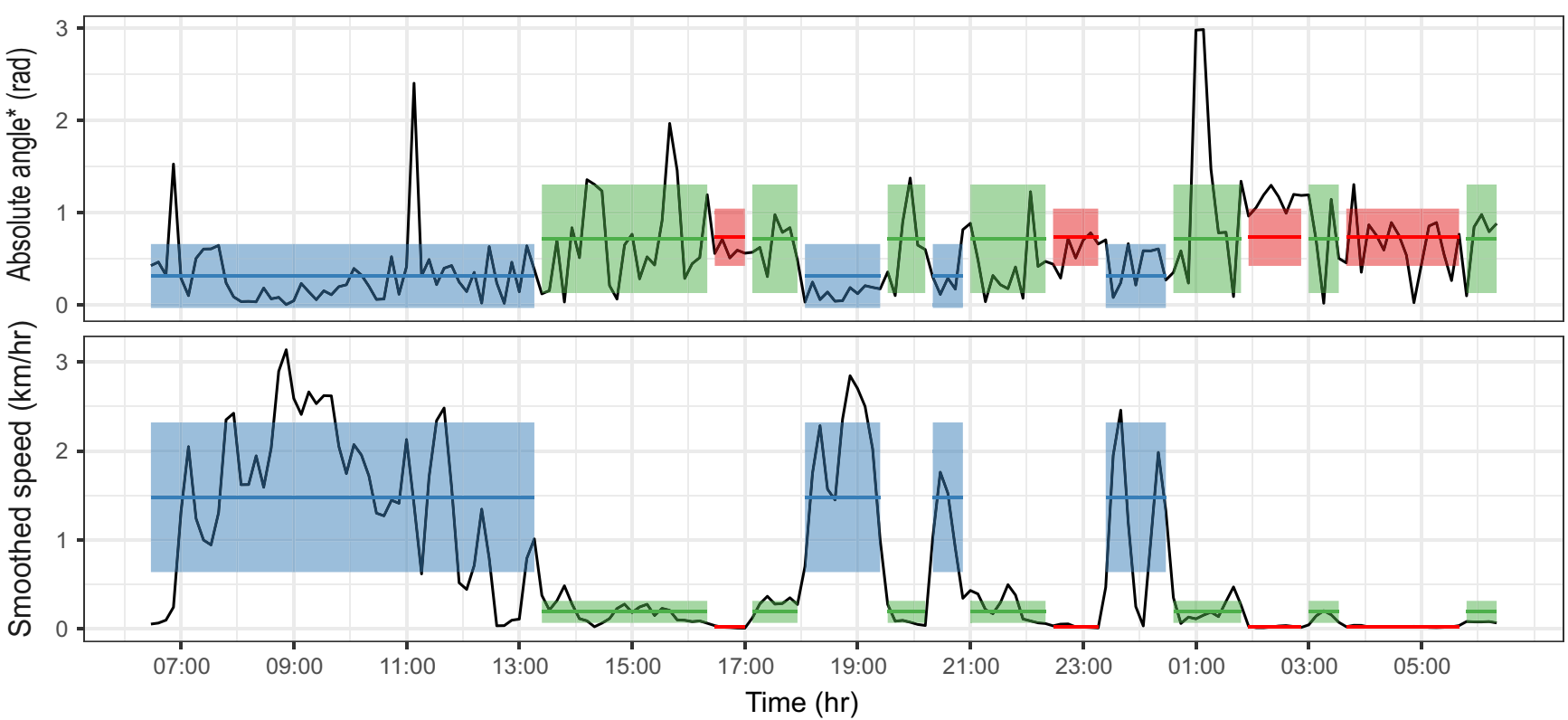

FIGURE 5 Example of application of the segclust2d/segmentation-clustering procedure to highlight behavioural changes in a 24-hr zebra's movement. (a) Path representation obtained by linking GPS locations recorded every 8 min; (b) determination using BIC-based penalized likelihood of the most likely numbers of states $(M=5)$ and segments $(K=20)$ (big orange dot), and most likely numbers of segments for the other number of states considered (squares at the top of the curves), with $L_{\min }=5$ (i.e. $40 \mathrm{~min}$ ); (c) corresponding time series in terms of absolute turning angle computed with a constant step length, $\left|\alpha_{i}^{*}\right|$, and smoothed speed, $\left(L_{i}+L_{i+1}\right) /(2 \Delta t)$, segmented with $M=3$ (leading to $K=15$ ); the coloured bands appearing over the two time series show the estimated occurrences and mean (plain horizontal line running in the middle of the band) $\pm S D$ (band width) for each of the three clusters considered

to understand how the occurrences and durations of home ranges are related to environmental co-variates, which is a prerequisite to infer long-term consequences for population distribution (Mueller \& Fagan, 2008). The elephant illustrative example also shows that, although the model underlying segclust $2 \mathrm{~d}$ looks for stationary phases, there is no guarantee that all segments obtained are really stationary. This occurs because changes of stationary phases are modelled as breakpoints but may in fact correspond to slow progressive changes.

Since the pioneering paper by Morales et al. (2004), HMMbased methods have often been considered the best way to detect changes of behavioural modes of remotely tracked animals. An alternative approach was proposed by Barraquand and Benhamou (2008). It consisted in computing the series of residence time within a virtual circle running along the path and to search for the most likely breakpoints using Lavielle (2005)'s univariate segmentation method. However, although the residence time provides a simple and reliable univariate signal easy to segment and interpret, the values obtained depend not only on the type of behaviour that is performed but also on how long it is performed, preventing the segments corresponding to the same behaviour from being easily 
clustered. In the present study, we show using computer simulations that the segclust $2 \mathrm{~d} / \mathrm{segmentation}$-clustering procedure rivals (and can even outperform) a HMM-based method initialized with state-dependent probability distribution parameters tuned to their true values for the different behavioural modes. With actual data, as the true values of parameters are usually unknown, our method, which does not require any initial guess, should have a clear advantage over the HMM-based method. It is worth noting that the best results were obtained with the joint use of metrics other than linear and angular speeds, such as smoothed speed and turning angle measured at constant step length, which were expected to improve the contrast between the intensive and extensive searching modes. Interestingly, using absolute rather than signed values of turning angles measured at constant step length works at best with our method, whereas right and left turns were balanced in any mode in our simulated movements. Such metrics should be particularly useful to distinguish between intensive and extensive modes when the former involves turning systematically right or left, that is characterized by markedly either negative or positive mean turning angles, whereas the latter involves balanced turning, as occurs in some species (e.g. Smith, 1974). As it results also in a reliable identification when turns are balanced in both intensive and extensive searching, we recommend using it systematically when using our method to distinguish between extensive and intensive searching phases.

In the illustrative example on zebra's movements, five behavioural modes were detected by the segclust2d/segmentationclustering procedure in the time series of smoothed speed and absolute value of the turning angles measured at constant step length. Nevertheless, based on behavioural observations, we chose to segment the time series with only three modes assumed to correspond to immobility, feeding and transit. Indeed, although our method can estimate the number of states based on a statistical criterion, it turns out in the computer simulations that this number was poorly estimated, despite the behavioural modes were clearly defined in this case. With actual data, there can be some mixing between modes, for instance transit and opportunistic feeding at some times, so that the estimation of the number of relevant modes may become unreliable. Thus, we recommend using the capacity of the segclust $2 \mathrm{~d} /$ segmentation-clustering procedure to estimate the number of states only when this number cannot be fixed a priori based on biological arguments. A similar conclusion was reached by Pohle et al. (2017) for HMM-based methods. It is also worth noting that feeding and resting can be distinguished based on movement characteristics only in animals which have to move significantly (with respect to the location recording noise) to feed. For animals which feed mainly without markedly moving, such as some browser herbivores and carcass-eating carnivores, ancillary activity data, such as those provided by on-board accelerometers, are required to distinguish these two behavioural modes. As our method can work conjointly on any number of time series of any nature, future implementation could integrate activity (accelerometer-based) time series for a better identification of resting versus active phases.
The segmentation of piecewise stationary time series, possibly complemented by the clustering of the resulting segments into functional classes, is key to understanding the dynamics of underlying processes. Based on bivariate time series of metrics such as easting and northing (home range shift studies), or speed and turning angles (movement mode studies), segclust2d has the potential to facilitate discovery in the field of movement ecology (e.g. Thaker, Gupte, Prins, Slotow, \& Vanak, 2019; Benoit et al. 2019). It is worth noting that this approach is not restricted to studies of home range shifts or of changes of movement modes. For example, the segmentation-only procedure can be used for highlighting marked changes in moving direction by segmenting the joint series of corresponding cosines and sines. If necessary, this approach can potentially apply to more dimensions as well, so as to consider ancillary variables such as activity and other metrics such as distance to a nest, proxies of habitat quality or any other variable that may be relevant when studying animal movements. As it can deal with two or more variables of any nature, our approach should be useful not only in movement ecology but also in many other fields, using appropriate metrics.

A theoretical limitation of our approach is that the metrics used are assumed to result in Gaussian and independent (for a given segment) values. The normality assumption makes computations easier but other distributions can be considered (Cleynen, Dudoit, \& Robin, 2014). For segmentation-only, deviation of the data from normality is not problematic, as the cost of additional segments, based on the log-likelihood when assuming normality, can be interpreted more generally (i.e. for any distribution) as a contrast based on mean and variance. For segmentation-clustering, the way deviation from normality may affect the results remains unclear. Furthermore, dynamic programming cannot be directly applied when some dependence is considered in this framework, even if some possibilities have recently emerged (Chakar, Lebarbier, Lévy-Leduc, \& Robin, 2017). However, computer simulations showed that our method is quite robust to violations of these assumptions. Thus, in home range shift study, despite northings and eastings showed a large serial correlation (even after subsampling; see Figure 1a,b) and were not normally distributed, our method was nevertheless able to correctly detect the small shift in mean location in $98 \%$ of replicates. Our method was also able to perform an efficient segmentation-clustering in the movement mode study, despite a serial correlation was mechanically introduced when considering the smoothed rather than usual speed, and the speed (drawn from a log-normal distribution) and the turns obtained for resting phases (and for any phase when turns were taken in absolute value) were not normally distributed. Some mathematical transformations may be used to attempt to normalize the data. However, using a log-transformed speed, which should a priori look like more Gaussian, did not improve the segmentation (results not shown), probably because the smoothing over two steps as well as the noise that affects the locations tend to distort the initial distribution. The choice of the metrics used should therefore first favour those that appear as being the easiest to interpret in terms of biological significance and the most able to give birth to piecewise stationary time series (i.e. the most likely to reveal the occurrence of a breakpoint 
between two successive phases). The question of normality and independence certainly matters, but to a lesser extent. For home range shift studies, northings and eastings appear to be the obvious choice. For movement mode studies, the smoothed speed and the absolute value of turns at constant step length, which characterize the movement in terms of temporal and spatial component separately, and seem able to maximize the contrast between the various modes when the recording noise is high, are certainly the two metrics to consider first.

\section{ACKNOWLEDGEMENTS}

We thank Clément Calenge and Fred Barraquand for interesting discussions about segmentation and clustering issues, and two anonymous reviewers for their constructive critiques. The study was partially funded by the Grant ANR-16-CE02-0001-01 of the French 'Agence Nationale de la Recherche', and the Zone Atelier program of the CNRS. Computer simulations were programmed in Pascal and ran thanks to the FreePascal compiler (www.freepascal.org).

\section{AUTHORS' CONTRIBUTIONS}

R.P. analysed the data and contributed to the coding of the statistical model, which was developed by M.-P.E. and E.L. S.C.-J. provided the tracking data. S.B. led the project, performed computer simulations and wrote the first draft of the manuscript, except the part describing the model which was first contributed to by M.-P.E., E.L. and R.P. All authors contributed significantly to the final manuscript.

\section{DATA AVAILABILITY STATEMENT}

The code of the method is publicly available as an $\mathrm{R}$ package (cran.rproject.org/package=segclust2d). The data used in the examples (24-hr GPS track of a plains zebra and GPS track of an African elephant recorded for $>2.5$ years) are available on Dryad: https://doi. org/10.5061/dryad.2j63369 (Patin, Etienne, Lebarbier, ChamailléJammes, \& Benhamou, 2019).

\section{ORCID}

Simon Chamaillé-Jammes (iD https://orcid.org/0000-0003-0505-6620 Simon Benhamou (iD https://orcid.org/0000-0003-0803-8559

\section{REFERENCES}

Barraquand, F., \& Benhamou, S. (2008). Animal movements in heterogeneous landscapes: Identifying profitable places and homogeneous movement bouts. Ecology, 89, 3336-3348. https://doi. org/10.1890/08-0162.1

Bellman, R. (1954). The theory of dynamic programming. Bulletin of the American Mathematical Society, 60, 503-516. https://doi. org/10.1090/S0002-9904-1954-09848-8
Benhamou, S. (1989). An olfactory orientation model for mammals' movements in their home ranges. Journal of Theoretical Biology, 139, 379-388. https://doi.org/10.1016/S0022-5193(89)80216-4

Benhamou, S. (2014). Of scales and stationarity in animal movements. Ecology Letters, 17, 261-272. https://doi.org/10.1111/ele.12225

Benhamou, S., \& Bovet, P. (1989). How animals use their environment: A new look at kinesis. Animal Behaviour, 38, 375-383. https://doi. org/10.1016/S0003-3472(89)80030-2

Benoit, L., Hewison, A. J. M., Coulon, A., Debeffe, L., Grémillet, D., Ducros, D., ... Morellet, N. (2020). Accelerating across the landscape: The energetic costs of natal dispersal in a large herbivore. Journal of Animal Ecology, 89, 173-185. https://doi.org/10.1111/ 1365-2656.13098

Beyer, H. L., Morales, J. M., Murray, D., \& Fortin, M. J. (2013). The effectiveness of Bayesian state space models for estimating behavioural states from movement paths. Methods in Ecology and Evolution, 4, 433-441. https://doi.org/10.1111/2041-210X.12026

Blackwell, P.G. (1997). Random diffusion models for animal movement. Ecological Modelling, 100, 87-102. https://doi.org/10.1016/ S0304-3800(97)00153-1

Breed, G. A., Golson, E. A., \& Tinker, M. T. (2017). Predicting animal home-range structure and transitions using a multistate OrnsteinUhlenbeck biased random walk. Ecology, 98, 32-47. https://doi. org/10.1002/ecy.1615

Bunnefeld, N., Börger, L., van Moorter, B., Rolandsen, C. M., Dettki, H., Solberg, E. J., \& Ericsson, G. (2011). A model-driven approach to quantify migration patterns: Individual, regional and yearly differences. Journal of Animal Ecology, 80, 466-476. https://doi. org/10.1111/j.1365-2656.2010.01776.x

Cagnacci, F., Focardi, S., Ghisla, A., van Moorter, B., Merrill, E. H., Gurarie, E., ... Hebblewhite, M. (2016). How many routes lead to migration? Comparison of methods to assess and characterize migratory movements. Journal of Animal Ecology, 85, 54-68. https://doi. org/10.1111/1365-2656.12449

Chakar, S., Lebarbier, E., Lévy-Leduc, C., \& Robin, S. (2017). A robust approach to multiple change-point estimation in an $A R(1)$ process. Bernoulli, 23, 1408-1447.

Cleynen, A., Dudoit, S., \& Robin, S. (2014). Comparing segmentation methods for genome annotation based on RNA-Seq data. Journal of Agricultural, Biological and Environmental Statistics, 19, 101-118. https ://doi.org/10.1007/s13253-013-0159-5

Couriot, O., Hewison, A. J. M., Saïd, S., Cagnacci, F., Chamaillé-Jammes, S., Linnell, J. D. C., ... Morellet, N. (2018). Truly sedentary? The multirange tactic as a response to resource heterogeneity and unpredictability in a large herbivore. Oecologia, 187, 47-60. https://doi. org/10.1007/s00442-018-4131-5

Dempster, A. P., Laird, N. M., \& Rubin, D. B. (1977). Maximum likelihood from incomplete data via the EM algorithm. Journal of the Royal Statistical Society B, 39, 1-38.

Dias, M. P., Granadeiro, J. P., \& Palmeirim, J. M. (2009). Searching behaviour of foraging waders: Does feeding success influence their walking? Animal Behaviour, 77, 1203-1209. https://doi.org/10.1016/j. anbehav.2009.02.002

Edelhoff, H., Signer, J., \& Balkenhol, N. (2016). Path segmentation for beginners: An overview of current methods for detecting changes in animal movement patterns. Movement Ecology, 4, 21.

Gloaguen, P., Mahévas, S., Rivot, E., Woillez, M., Guitton, J., Vermard, Y., \& Etienne, M.-P. (2015). An autoregressive model to describe fishing vessel movement and activity. Environmetrics, 26, 17-28. https://doi. org/10.1002/env.2319

Gurarie, E., Andrews, R. D., \& Laidre, K. L. (2009). A novel method for identifying behavioural changes in animal movement data. Ecology Letters, 12, 395-408. https://doi.org/10.1111/j.1461-0248.2009.01293.x

Gurarie, E., Bracis, C., Delgado, M., Meckley, T. D., Kojola, I., \& Wagner, C. M. (2016). What is the animal doing? Tools for exploring behavioural 
structure in animal movements. Journal of Animal Ecology, 85, 69-84. https://doi.org/10.1111/1365-2656.12379

Gurarie, E., Cagnacci, F., Fleming, C., Peters, W., Calabrese, J. M. Mueller, T., \& Fagan, W. F. (2017). A framework for modelling range shifts and migrations: Asking when, whither, whether and will it return. Journal of Animal Ecology, 86, 943-959. https://doi. org/10.1111/1365-2656.12674

Karlin, S., \& Taylor, H. M. (1975). A first course in stochastic processes. New York, NY: Academic Press.

Langrock, R., King, R., Matthiopoulos, J., Thomas, L., Fortin, D., \& Morales, J. M. (2012). Flexible and practical modeling of animal telemetry data: Hidden Markov models and extensions. Ecology, 93, 2336-2342. https://doi.org/10.1890/11-2241.1

Lavielle, M. (2005). Using penalized contrasts for the change-point problem. Signal Processing, 85, 1501-1510. https://doi.org/10.1016/j. sigpro.2005.01.012

Levin, S. A. (1992). The problem of pattern and scale in ecology. Ecology, 73, 1943-1967.

McClintock, B. T., King, R., Thomas, L., Matthiopoulos, J., McConnell, B. J., \& Morales, J. M. (2012). A general discrete-time modeling framework for animal movement using multistate random walks. Ecological Monographs, 82, 335-349. https://doi.org/10.1890/11-0326.1

McClintock, B. T., \& Michelot, T. (2018). momentuHMM: R package for generalized hidden Markov models of animal movement. Methods in Ecology and Evolution, 9, 1518-1530. https://doi. org/10.1111/2041-210X.12995

Michelot, T., \& Blackwell, P. G. (2019). State-switching continuous-time correlated random walks. Methods in Ecology and Evolution, 10, 637649. https://doi.org/10.1111/2041-210X.13154

Michelot, T., Langrock, R., \& Patterson, T. A. (2016). moveHMM: An $\mathrm{R}$ package for the statistical modelling of animal movement data using hidden Markov models. Methods in Ecology and Evolution, 7, 1308-1315.

Monsarrat, S., Benhamou, S., Sarrazin, F., Bessa-Gomes, C., Bouten, W., \& Duriez, O. (2013). How predictability of feeding patches affects home range and foraging habitat selection in avian social scavengers? PLOS ONE, 8, e53077. https://doi.org/10.1371/journ al.pone.0053077

Morales, J. M., Haydon, D. T., Frair, J., Holsinger, K. E., \& Fryxell, J. M. (2004). Extracting more out of relocation data: Building movement models as mixtures of random walks. Ecology, 85, 2436-2445. https ://doi.org/10.1890/03-0269

Mueller, T., \& Fagan, W. F. (2008). Search and navigation in dynamic environments from individual behaviors to population distributions. Oikos, 117, 654-664. https://doi.org/10.1111/j.0030-1299.2008.16291.x

Naidoo, R., Du Preez, P., Stuart-Hill, G., Jago, M., \& Wegmann, M. (2012). Home on the range: Factors explaining partial migration of African buffalo in a tropical environment. PLoS ONE, 7, e36527.
Nams, V. O. (2014). Combining animal movements and behavioural data to detect behavioural states. Ecology Letters, 17, 1228-1237. https:// doi.org/10.1111/ele.12328

Parton, A., \& Blackwell, P. G. (2017). Bayesian inference for multistate 'Step and Turn' animal movement in continuous time. Journal of Agricultural, Biological and Environmental Statistics, 22, 373-392. https://doi.org/10.1007/s13253-017-0286-5

Patin, R., Etienne, M. P., Lebarbier, E., Chamaillé-Jammes, S., \& Benhamou, S. (2019). Data from: Identifying stationary phases in multivariate time-series for highlighting behavioural modes and home range settlements. Dryad Digital Repository, https://doi.org/10.5061/ dryad.2j63369

Picard, F., Robin, S., Lebarbier, E., \& Daudin, J.-J. (2007). A segmentation/ clustering model for the analysis of array CGH data. Biometrics, 63, 758-766. https://doi.org/10.1111/j.1541-0420.2006.00729.x

Pohle, J., Langrock, R., van Beest, F. M., \& Schmidt, N. M. (2017). Selecting the number of states in hidden Markov models: Pragmatic solutions illustrated using animal movement. Journal of Agricultural, Biological and Environmental Statistics, 22, 270-293. https://doi.org/10.1007/ s13253-017-0283-8

Pyke, G. H. (1978). Are animals efficient harvesters? Animal Behaviour, 26, 241-250. https://doi.org/10.1016/0003-3472(78)90024-6

Rigaill, G. (2015). A pruned dynamic programming algorithm to recover the best segmentations with 1 to kmax change-points. Journal De La Société Française De Statistique, 156, 180-205.

Schwarz, G. (1978). Estimating the dimension of a model. The Annals of Statistics, 6, 461-464. https://doi.org/10.1214/aos/1176344136

Smith, J. N. (1974). The food searching behaviour of two European thrushes. II. The Adaptiveness of Search Patterns. Behaviour, 49, 1-61.

Thaker, M., Gupte, P. R., Prins, H. H., Slotow, R., \& Vanak, A. (2019). Finescale tracking of ambient temperature and movement reveals shuttling behavior of elephants to water. Frontiers in Ecology and Evolution, 7, 4.

\section{SUPPORTING INFORMATION}

Additional supporting information may be found online in the Supporting Information section at the end of the article.

How to cite this article: Patin R, Etienne M-P, Lebarbier E, Chamaillé-Jammes $\mathrm{S}$, Benhamou S. Identifying stationary phases in multivariate time series for highlighting behavioural modes and home range settlements. J Anim Ecol. 2020;89:44-56. https://doi.org/10.1111/1365-2656.13105 